\title{
GeVIR is a continuous gene-level metric that uses variant distribution patterns to prioritize disease candidate genes
}

DOI:

10.1038/s41588-019-0560-2

\section{Document Version}

Accepted author manuscript

Link to publication record in Manchester Research Explorer

\section{Citation for published version (APA):}

Abramovs, N., Brass, A., \& Tassabehji, M. (2020). GeVIR is a continuous gene-level metric that uses variant distribution patterns to prioritize disease candidate genes. Nature Genetics, 52(1), 35-39.

https://doi.org/10.1038/s41588-019-0560-2

\section{Published in:}

Nature Genetics

\section{Citing this paper}

Please note that where the full-text provided on Manchester Research Explorer is the Author Accepted Manuscript or Proof version this may differ from the final Published version. If citing, it is advised that you check and use the publisher's definitive version.

\section{General rights}

Copyright and moral rights for the publications made accessible in the Research Explorer are retained by the authors and/or other copyright owners and it is a condition of accessing publications that users recognise and abide by the legal requirements associated with these rights.

\section{Takedown policy}

If you believe that this document breaches copyright please refer to the University of Manchester's Takedown Procedures [http://man.ac.uk/04Y6Bo] or contact uml.scholarlycommunications@manchester.ac.uk providing relevant details, so we can investigate your claim.

\section{OPEN ACCESS}


2 GeVIR is a continuous gene-level metric that uses variant distribution patterns to

3 prioritize disease candidate genes

4

5 Nikita Abramovs ${ }^{1,2}$, Andrew Brass ${ }^{1,3}$, and May Tassabehji ${ }^{2,4 *}$

6

$7 \quad{ }^{1}$ School of Computer Science, University of Manchester, Manchester, UK.

$8{ }^{2}$ School of Biological Sciences, Faculty of Biology, Medicine and Health, University of Manchester,

9 Manchester, UK.

$10{ }^{3}$ School of Health Sciences, Faculty of Biology, Medicine and Health, University of Manchester,

11 Manchester, UK.

$12{ }^{4}$ Manchester Centre for Genomic Medicine, St. Mary’s Hospital, Manchester Academic Health

13 Sciences Centre (MAHSC), Manchester, UK.

14

$15 *$ e-mail: m.tassabehji@manchester.ac.uk 
With large scale population sequencing projects gathering pace, there is a need for strategies that advance disease gene prioritization ${ }^{1,2}$. Metrics that provide information about a gene and its ability to tolerate protein-altering variation can aid clinical interpretation of human genomes and advance disease gene discovery ${ }^{1-4}$. Previous methods analyzed total variant load in a gene ${ }^{1-4}$, but not their distribution pattern within a gene. Using data from 138,632 exome/genome sequences ${ }^{2}$, we developed Gene Variation Intolerance Rank (GeVIR) to produce a continuous gene-level metric for 19,361 genes that is able to prioritize both dominant and recessive Mendelian disease genes $^{5}$, outperforming missense constraint metrics ${ }^{3}$ and comparable, but complementary, to lossof-function (LoF) constraint metrics ${ }^{2}$. GeVIR is also able to prioritize short genes, for which LoF constraint cannot be confidently estimated ${ }^{2}$. A majority of the most intolerant genes identified here have no defined phenotype and are candidates for severe dominant disorders.

The increasing size of publicly available variant databases from large predominantly healthy populations ${ }^{1,2}$ provides us with an intriguing opportunity to analyze small gene-coding regions devoid of variants, as an alternative to existing methods that measure variant deficiency at a gene level ${ }^{1-4,6}$ or within large coding regions ${ }^{7,8}$. Since some disease-causing variants are dominant, highly penetrant, and tend to cluster in functionally important coding regions ${ }^{9}$, it is reasonable to hypothesize that genes important for human development might have regions that are so sensitive to variation they are never observed in healthy individuals. These regions were recently studied in the context of variant prioritization $^{10}$; however, a continuous gene-level variation intolerance metric utilizing variant distribution was not developed.

To illustrate the hypothesis behind GeVIR, which is based on analyzing the length, evolutionary conservation, and number of Variant Intolerant Regions (VIR, a region between two protein-altering variants) in gnomAD (v2.0.1), we analyzed 3.5 million VIRs in 19,361 protein-coding genes and 
41 ClinVar ${ }^{11}$. Although $\sim 91 \%$ of the VIRs were small (1-5 amino acids), only $\sim 53 \%$ of pathogenic

missense variants were located within them (Fig. 1a,b). Moreover, pathogenic missense variants were observed $\sim 3.7$ times (two-sided Fisher's exact test $P<2.2 \times 10^{-308}$, Supplementary Table 1 ) more often inside the largest VIRs ( $>20$ amino acids), a trend that was not observed for LoF variants (Fig. 1c).

There was also a positive correlation between VIR length and evolutionary conservation, measured by mean GERP++ score ${ }^{12}$ among 1,938,102 VIRs (length $\geq 1$ amino acid) in 18,491 genes (Fig. 1d).

To create GeVIR, we counted the number of VIRs of each length (only with high coverage) in the canonical transcripts of 19,361 genes. Examples of variant distribution patterns in four disease genes with different modes of inheritance and length are demonstrated in Figure 2a. We calculated the weight of each VIR length based on how frequently it was observed among all genes (Fig. 2b-d). A score for each gene was calculated as a sum of all its VIR weights, multiplied by average GERP++ score and divided by the total number of VIRs in a gene (including those with low coverage) (Fig. 2e). High evolutionary conservation supported region importance and increased the weight, whereas low conservation was penalized because lack of variation could be a result of sequencing or variant filtering errors (scores between -1 and 1 were rounded to avoid extreme penalties from multiplication). Finally, genes were sorted using this score to create GeVIR percentiles (Supplementary Table 2).

To evaluate GeVIR performance, we compared its ability to rank genes that are expected to have different levels of intolerance to variation with recently released gnomAD gene constraint metrics: Missense z-score ${ }^{3}$, missense and loss-of-function observed/expected upper bound fraction called MOEUF and LOEUF (a recommended replacement for the probability of being LoF intolerant score, $\mathrm{pLI})^{2}$, respectively (Table 1). GeVIR outperformed both missense metrics at prioritizing autosomal dominant $(\mathrm{AD})^{5}$, human orthologs of mouse heterozygous lethal knockouts ${ }^{13,14}$, and cell essential ${ }^{15}$ genes by $\sim 4-6 \%$ AUC, whereas LOEUF showed a similar performance to GeVIR in the first and the second assays, but was $\sim 4 \%$ lower in the last assay. GeVIR ranked genes with homozygous LoF variants in healthy populations (Null) ${ }^{1}$ and cell non-essential ${ }^{15}$ genes $\sim 2 \%$ less effectively than 
Missense z-scores, which achieved the best results in both assays. Importantly, however, GeVIR and LOEUF showed a much higher trend to prioritize autosomal recessive $(\mathrm{AR})^{5}$ genes closer to the middle of the ranked list ( 1.7-1.8 fold enrichment (FE) at the $40^{\text {th }}-60^{\text {th }}$ percentiles) than missense metrics (Missense z-scores $\sim 1.1 \mathrm{FE}, P=0.015$; MOEUF $\sim 1.3 \mathrm{FE}, P=8.86 \times 10^{-7}$; Fig $3 \mathrm{a}$ and Supplementary Table 3). Considering that Null and cell non-essential (i.e. low intolerant) genes could also be disease causing (Supplementary Table 4) and from results of previous assays (Table 1), the distribution of AR genes showed that GeVIR and LOEUF outperformed missense metrics in prioritizing essential and disease genes. A good ranking method should also prioritize highly intolerant (e.g. AD) over medium intolerant (e.g. AR) genes; therefore, the performance of all metrics at distinguishing AD (true positive) from AR (false positive) genes was evaluated by calculating F1 scores for the AD class cumulatively at each percentile. GeVIR achieved a $62.3 \%$ peak F1 score and surpassed missense metrics by $\sim 2-3 \%$, but was outperformed by LOEUF by $\sim 2 \%$.

To clarify the impact of evolutionary conservation on GeVIR, scores were recalculated without GERP++ adjustments and assessed in the same assays (Table 1). Despite a decreased performance, it still outperformed missense metrics in prioritizing essential and disease genes, but performed worse in ranking Null and cell non-essential genes, which probably had shorter VIRs and consequently relied more on GERP++ adjustments. Nevertheless, this highlights that, for estimating missense intolerance, GeVIR is a better alternative to state-of-the-art methods that are based on observed/expected ratios of $\operatorname{variants}^{2,3}$.

Although GeVIR and LOEUF showed a comparable performance and, unlike missense metrics, demonstrated a potential to rank AR genes, they represent gene intolerance to different types of variation (i.e. missense and LoF, respectively). Moreover, LOEUF was significantly more biased towards longer genes (Spearman $r=-0.54)$ than GeVIR $(r=-0.26)$, especially in the first decile (median protein length $\sim 1.91$ and $\sim 1.15$ times longer than expected 425 amino acids, respectively) (Fig. 3b). To rank genes by intolerance to both missense and LoF, GeVIR and LOEUF were combined into 
VIRLoF (rank summation with re-sorting), which resulted in a more optimal ranking that outperformed other metrics in most assays (Table 1).

To investigate this difference, we selected 2,989 genes ( 15.4\%) with the lowest LOEUF $(<0.35$; a recommended hard threshold for most intolerant genes; L. Francioli, G. Tiao, K. Karczewski, M. Solomonson and N. Watts from https://macarthurlab.org/2018/10/17/gnomad-v2-1/), and the same number of genes ranked as the most intolerant by GeVIR and VIRLoF. At this threshold, GeVIR and LOEUF showed a similar performance $(\mathrm{F} 1=\sim 56.5 \%)$ and were outperformed by VIRLoF $(\mathrm{F} 1=$ $\sim 60.2 \%)$; however, only $\sim 55.9 \%(1,637)$ of the genes prioritized by GeVIR were the same as those prioritized by LOEUF (Fig. 4a,d), highlighting a need for both metrics. This group of both missense and LoF most intolerant genes contained $\sim 31.7 \%$ (250/790) of known AD genes, and only $\sim 2.2 \%$ $(35 / 1,585)$ of known AR genes ( 14.3 fold enrichment, two-sided Fisher's exact test $\left.P=1.0 \times 10^{-72}\right)$ (Fig. $4 \mathrm{~b}, \mathrm{c})$. Moreover $\sim 70 \%$ of these genes $(1,164 / 1,637)$ are not yet linked to any phenotype in $\mathrm{OMIM}^{5}$, but they are significantly enriched $\left(\sim 3.8 \mathrm{FE}, P=7.9 \times 10^{-10}\right)$ with mouse heterozygous lethal genes $(36 / 1,164)$ compared with unknown genes in the remaining $\sim 91.4 \%(115 / 14,174)$. This suggests that they might be crucial for organism development, and heterozygous de novo deleterious variants in them are likely to result in pathogenic phenotypes.

Missense and LoF intolerant genes $(1,637)$ were enriched in histone modification and dopaminergic synapse function, a trend not observed with genes prioritized by either GeVIR or LOEUF alone (“functional annotation term” enrichment analysis performed using DAVID v6.8 $8^{16,17}$, Supplementary Note and Supplementary Tables 5 and 6$)$. Genes prioritized only by GeVIR (1,317; i.e. intolerant to missense variants) were enriched in spliceosome complex function, similar to those prioritized by both metrics $(1,672)$, but the former were generally shorter, with 221 and 559 amino acid median protein length, respectively. This trend was not observed with genes prioritized only by LOEUF and highlights that potentially important short genes could be missed without GeVIR, possibly due to an insufficient gnomAD population size, an acknowledged limitation of LOEUF ${ }^{2}$. 
Although GeVIR is based on the same principle as the Conservative Coding Regions (CCR)

$117 \operatorname{map}^{10}$ (i.e. analyzed distance between variants in gnomAD), it is important to differentiate between

118 gene (GeVIR) and variant (a potential application of CCR) prioritization methods. For example,

119 TARDBP, associated with AD diseases, is among the top $1 \%$ of the genes ranked by GeVIR since it

120 contained many long VIRs, but all associated ClinVar pathogenic variants would be misclassified by

121 CCR since they are located outside long CCRs/VIRs (Fig. 2a). Havrilla et al. ${ }^{10}$ compared their method

122 with other gene constraint metrics by ranking genes based on the number of CCRs $\geq 95 \%$. To allow

123 comparisons, we used the same approach (7,000 genes with at least one CCR $\geq 95 \%$ and a GeVIR

124 rank) and found that GeVIR, even without GERP++ adjustments, was less biased by gene length

125 (Spearman $r=0.03 / 0.04)$ and more efficiently distinguished AD from AR genes (peak F1 =

$12662.38 / 61.68 \%)$, than CCR $(r=-0.29, \mathrm{~F} 1=57.25 \%)($ Extended Data Fig. 1). Moreover, GeVIR provides

127 a continuous metric of intolerance for 19,361 genes that can be used for AR gene prioritization,

128 whereas CCR is limited to $\sim 7,000$ genes and therefore can only be used for AD gene/variant

129 prioritization $^{10}$.

130 Here we present a novel method to measure gene variation intolerance by analyzing variant

131 distribution patterns within genes. GeVIR mostly represents gene intolerance to missense variation,

132 outperforming existing gnomAD missense gene constraint metrics and complementing the LoF metric,

133 due to its ability to estimate intolerance of short genes (e.g. LITAF, associated with AD Charcot-Marie-

134 Tooth disease, length $=162$ amino acids, ranked by GeVIR $\sim 35 \%$, but by gnomAD metrics $\sim 67-72 \%$,

135 Fig. 2a). GeVIR, like other gene constraint metrics derived from gnomAD data, might underestimate

136 intolerance of genes associated with phenotypes that could be present in the gnomAD population (e.g.

137 late onset diseases, cancer) ${ }^{18}$, but is not biased towards more studied genes (a criticism associated with

138 gene scores that rely on gene networks ${ }^{19,20}$ ). GeVIR scores are available online (www.gevirank.org),

139 with additional metrics to facilitate prediction of the inheritance mode (AD/AR) for potential disease 
140 genes (Supplementary Note and Supplementary Fig. 5), and should aid interpretation of genome

141 sequencing data in a clinical setting and advance human disease gene discovery.

142

\section{Acknowledgments}

144 This work was supported by the Engineering and Physical Sciences Research Council [EP/N509565/1].

145 M.T. was funded by the Newlife Foundation [grant \#14-15/15]. We also acknowledge the support of

146 the Manchester Academic Health Science Centre. We thank the Genome Aggregation Database

147 (gnomAD) and the groups that provided exome and genome variant data to this resource. A full list of

148 contributing groups can be found at https://gnomad.broadinstitute.org/about.

149

150 Author Contributions

151 N.A., M.T., and A.B. conceived and designed the research. N.A. executed the analysis. N.A. and M.T.

152 performed the primary writing. M.T. and A.B. supervised all aspects of the research, and reviewed and

153 edited the manuscript.

154

155 Competing interests

156 The authors declare no competing interests.

157 
1. Lek, M. et al. Analysis of protein-coding genetic variation in 60,706 humans. Nature 536, 285-291 (2016).

2. Karczewski, K. J. et al. Variation across 141,456 human exomes and genomes reveals the spectrum of loss-of-function intolerance across human protein-coding genes. bioRxiv 531210 (2019) doi:10.1101/531210.

3. Samocha, K. E. et al. A framework for the interpretation of de novo mutation in human disease. Nat. Genet. 46, 944-950 (2014).

4. Petrovski, S., Wang, Q., Heinzen, E. L., Allen, A. S. \& Goldstein, D. B. Genic intolerance to functional variation and the interpretation of personal genomes. PLoS Genet. 9, e1003709 (2013).

5. Hamosh, A., Scott, A. F., Amberger, J. S., Bocchini, C. A. \& McKusick, V. A. Online Mendelian Inheritance in Man (OMIM), a knowledgebase of human genes and genetic disorders. Nucleic Acids Res. 33, D514-D517 (2005).

6. Cassa, C. A. et al. Estimating the selective effects of heterozygous protein-truncating variants from human exome data. Nat. Genet. 49, 806-810 (2017).

7. Gussow, A. B., Petrovski, S., Wang, Q., Allen, A. S. \& Goldstein, D. B. The intolerance to functional genetic variation of protein domains predicts the localization of pathogenic mutations within genes. Genome Biol. 17, 9 (2016).

8. Samocha, K. E. et al. Regional missense constraint improves variant deleteriousness prediction. bioRxiv 148353 (2017) doi:10.1101/148353.

9. Sivley, M. Comprehensive analysis of constraint on the spatial distribution of missense variants in human protein structures. Am. J. Hum. Genet. 102, 415-426 (2018).

10. Havrilla, J. M., Pedersen, B. S., Layer, R. M. \& Quinlan, A. R. A map of constrained coding regions in the human genome. Nat. Genet. 51, 88-95 (2018).

11. Landrum, M. J. et al. ClinVar: public archive of interpretations of clinically relevant variants. Nucleic Acids Res. 44, D862-D868 (2016).

12. Davydov, E. V. et al. Identifying a high fraction of the human genome to be under selective constraint using GERP++. PLoS Comput Biol 6, e1001025 (2010).

13. Motenko, H., Neuhauser, S. B., O’Keefe, M. \& Richardson, J. E. MouseMine: a new data warehouse for MGI. Mamm Genome 26, 325-330 (2015).

14. Eppig, J. T. et al. The Mouse Genome Database (MGD): facilitating mouse as a model for human biology and disease. Nucleic Acids Res. 43, D726-D736 (2015).

15. Hart, T. et al. Evaluation and design of genome-wide CRISPR/SpCas9 knockout screens. G3 7, 2719-2727 (2017).

16. Huang, D. W., Sherman, B. T. \& Lempicki, R. A. Systematic and integrative analysis of large gene lists using DAVID bioinformatics resources. Nat. Protoc. 4, 44-57 (2009).

17. Huang, D. W., Sherman, B. T. \& Lempicki, R. A. Bioinformatics enrichment tools: paths toward the comprehensive functional analysis of large gene lists. Nucleic Acids Res 37, 1-13 (2009).

18. Kobayashi, Y. et al. Pathogenic variant burden in the ExAC database: an empirical approach to evaluating population data for clinical variant interpretation. Genome Med. 9, 13 (2017).

19. Huang, N., Lee, I., Marcotte, E. M. \& Hurles, M. E. Characterising and predicting haploinsufficiency in the human genome. PLoS Genet 6, e1001154 (2010).

20. Steinberg, J., Honti, F., Meader, S. \& Webber, C. Haploinsufficiency predictions without study bias. Nucleic Acids Res. 43, e101-e101 (2015). 
160 Fig. 1 | Correlation between length of variant intolerant regions (VIRs), location of pathogenic 161 variants, and evolutionary conservation. a, Distances in amino acids between two non-synonymous variants (i.e. VIRs) from gnomAD in 2,935 genes containing at least one missense or $\mathrm{LoF}$ (stop gained or frameshift) pathogenic variant. b,c, Distribution of pathogenic missense and LoF variants inside VIRs (b) and normalized by cumulative region length (c). In $\mathbf{c}$, the central values represent pathogenic variants per amino acid ratio computed on 2,935 genes, whereas error bars represent $95 \%$ confidence interval from bootstapping ( $n=10,000$ iterations of resampling with replacement on $50 \%$ of 2,935 genes (i.e. $n=1,467$ ); each iteration result is shown on overlaid dot plots). $\mathbf{d}$, Correlation between VIR length and evolutionary conservation (GERP++ scores) of 1,938,102 VIRs with high coverage in 18,491 genes.

Fig. 2 | GeVIR workflow. a, Comparison of distribution of pathogenic missense, stop gained and frameshift variants from ClinVar with non-synonymous protein coding variants from gnomAD in 4 genes (TCF4, LITAF, ARSA and TARDBP) associated with Mendelian diseases. Variant Intolerant Regions (VIR) can be seen as "gaps" in the genes. b, Boundaries of nucleotide regions used to calculate VIR properties. c, Two genes (A and B) containing a total of 5 variants (orange boxes), which divide genes A and B into 4 and 3 VIRs, respectively, 6 of which are valid (5 shown in green and 1 (VIR4) with zero length). In gene B, VIR2 has low mean sequence coverage (48, shown in white) and is classified as non-valid. $\mathbf{d}$, Calculation of valid region length weights based on their frequency in all genes (longer regions are rare and have a larger weight). e, Gene score calculations for A and B: each valid region weight in a gene is multiplied by its GERP++ score and their sum is divided by the total number of regions in the gene (valid and non-valid). GeVIR ranking is based on these gene scores sorted in descending order. According to GeVIR, gene A is more intolerant to variants than gene B.

Fig. 3 Comparison of GeVIR gene ranking with gnomAD constraint metrics on 19,361 genes. a, Percentage of genes associated exclusively with autosomal recessive (AR) diseases out of all AR genes in OMIM $(n=1,585)$ in each rank decile. $\mathbf{b}$, Median protein length (amino acids) in each rank decile. Correlation between protein length and gene rank was measured with Spearman's rank correlation coefficient.

Fig. 4 | Comparison of GeVIR, LOEUF and VIRLoF performance on the most variant intolerant genes. a-c, 2,989/19,361 genes ( $15 \%)$ with upper observed/expected loss-of-function (LOEUF) scores $<0.35$ (green), and the same number of genes with the highest GeVIR (red) and VIRLoF (purple) scores. Venn diagrams show overlaps between all 2,989 genes (a), genes exclusively associated with autosomal dominant (AD) (b) or autosomal recessive (AR) (c) OMIM genes. d, Method performance is compared, considering $\mathrm{AD}$ genes as true positives and $\mathrm{AR}$ genes as false positives, using precision, recall and F1 metrics of AD class. 
Table 1 | Comparison of GeVIR gene ranking with gnomAD constraint metrics on 19,361 genes. Autosomal dominant (AD) and autosomal recessive (AR) gene groups consisted of genes associated with diseases with only one mode of inheritance in $\mathrm{OMIM}^{5}$. Human cell essential and non-essential gene groups were based on CRISPR/Cas screens ${ }^{15}$. Metrics performance was measured with area under the curve (AUC). Assays in which lower AUC indicates better performance are marked with *. In the $\mathrm{AD} / \mathrm{AR}$ classification assay, $\mathrm{AD}$ class $\mathrm{F} 1$ score is calculated at each percentile (cumulative) considering $\mathrm{AD}$ genes as true positives and $\mathrm{AR}$ genes as false positives and performance is reported as peak F1 score with gene percentile in brackets. Results of metrics that outperformed others were highlighted in bold. If VIRLoF showed the best results, then the second best performing metric was also highlighted in bold.

\begin{tabular}{|c|c|c|c|c|c|c|c|}
\hline Assay & Gene number & GeVIR & $\begin{array}{c}\text { GeVIR } \\
\text { (without GERP++) }\end{array}$ & $\begin{array}{c}\text { Missense } \\
\text { z-scores }\end{array}$ & MOEUF & LOEUF & VIRLoF \\
\hline $\begin{array}{c}\text { Autosomal dominant } \\
\text { (AD) }\end{array}$ & 790 & $\mathbf{7 3 . 5 4}$ & 72.80 & 68.72 & 68.21 & 72.40 & $\mathbf{7 4 . 3 8}$ \\
\hline $\begin{array}{c}\text { Mouse heterozygote } \\
\text { lethal knockout }\end{array}$ & 388 & 74.92 & 74.10 & 69.71 & 69.45 & $\mathbf{7 6 . 0 3}$ & $\mathbf{7 7 . 1 8}$ \\
\hline Cell essential & 663 & $\mathbf{7 2 . 6 2}$ & 69.97 & 66.81 & 68.22 & 68.70 & 72.13 \\
\hline Nulls* & 328 & 27.10 & 32.88 & $\mathbf{2 4 . 6 9}$ & 27.89 & 31.18 & 27.38 \\
\hline Cell non-essential* & 865 & 29.61 & 37.07 & $\mathbf{2 7 . 2 6}$ & 27.61 & 27.92 & $\mathbf{2 7 . 0 8}$ \\
\hline $\begin{array}{c}\text { AD/AR } \\
\text { classification }\end{array}$ & $\begin{array}{c}790 \mathrm{AD}, \\
1,585 \mathrm{AR}\end{array}$ & $\begin{array}{c}62.34 \\
(29.94)\end{array}$ & $61.48(25.44)$ & $\begin{array}{c}59.13 \\
(35.40)\end{array}$ & $\begin{array}{c}60.27 \\
(32.82)\end{array}$ & $\begin{array}{c}\mathbf{6 4 . 1 5} \\
\mathbf{( 2 7 . 1 7 )}\end{array}$ & $\begin{array}{c}\mathbf{6 5 . 7 4} \\
\mathbf{( 2 5 . 8 4 )}\end{array}$ \\
\hline
\end{tabular}


Online Methods

210 Protein-coding gene list. The gnomAD database (version 2.0.1) was used

211 (https://github.com/macarthur-lab/gnomad browser), which contained gene annotation from

212 GENCODE (version 19). Gene nucleotide and peptide sequences were obtained from Ensembl Biomart 213 (GRCh37/hg19) (http://grch37.ensembl.org/biomart/martview/) and gene constraint metrics from 214 gnomAD (https://gnomad.broadinstitute.org/). Only genes with canonical transcripts that start with 215 methonine, end with a stop codon, with protein coding nucleotide sequence divisible by 3 , and without 216 reported issues in gnomAD gene constrain metrics were used. The gene list consisted of 19,361 genes 217 (18,326 were linked with $\mathrm{HGNC}^{21}$ approved symbols by Ensembl IDs), 1,009 of which were marked as 218 outliers (e.g. "too many missense variants") in the gnomAD gene constraint metrics study ${ }^{2}$.

Variant Intolerant Regions (VIRs). Variants in canonical transcripts of 19,361 genes were analyzed by looking at regions between two variants (i.e. referred to here as start and stop of a VIR) predicted by Variant Effect Predictor (VEP, version 85) to be affecting protein amino acid sequence, namely: “stop_gained”, “frameshift_variant”, “stop_lost”, “start_lost”, “inframe_insertion”, "inframe_deletion”, “missense_variant". Only variants with filter status "PASS", "LCR" (low-complexity) or "SEGDUP" (segmental duplication) were analyzed. If start and stop variants were located in different exons, protein-coding nucleotides from multiple exons were concatenated into a single region. To take into account regions between a start codon and the first variant or the last variant and a stop codon in a gene, extra "fake" variants were added at the start and stop codons of all transcripts analyzed. We excluded 24,276 regions in which start or stop variant consequence predicted by VEP were inconsistent with transcript exon chromosome location (e.g. "missense" variant outside exon boundaries). The remaining dataset contained 4,323,481 regions, which we called Variant Intolerant Regions (VIRs). 
233 VIR properties. We measured three properties of VIR: length, mean coverage (gnomAD exomes) and 234 conservation (GERP $++^{12}$ ) (see Fig. $2 \mathrm{~b}$ and Supplementary Fig. 1). VIR length and conservation were 235 measured at the amino acid level. Regions between variants in two adjacent amino acids were included 236 in the analysis, but had zero length and conservation score. VIR coverage was measured as mean 237 exome coverage of nucleotides including those affected by region start and stop variants. Exome rather 238 than genome coverage was measured because the majority of individuals in gnomAD were exome 239 sequenced $(123,136$ out of 138,632$)$, and exome coverage tends to be less stable and thus could better 240 highlight potential bias in variant load. Since variant absence might be a consequence of low coverage, 241 strict filters were used to separate high and low covered VIRs. Autosomes, on average, had higher 242 coverage than allosomes and different coverage thresholds were used to mark approximately $80 \%$ of 243 the VIRs in both groups as high coverage: $\geq 50$ for autosomes $(3,427,010$ out of $4,221,872, \sim 81.17 \%)$ 244 and $\geq 45$ for allosomes $(82,033$ out of $101,609, \sim 80.73 \%)$. Note that $1,570,941$ VIRs were formed by variants in adjacent amino acids and therefore had zero length.

Pathogenic variants and evolutionary conservation of VIRs. Variants with known disease association status (e.g. "Pathogenic", "Likely pathogenic” or "Pathogenic/Likely pathogenic”) were downloaded from ClinVar ${ }^{11}$ (accessed August 2018) and re-annotated with Ensembl VEP (version 90). Only variants predicted by VEP to be "missense_variant” $(29,433)$, “stop_gained” $(15,991)$ or “frameshift_variant” (21,471), which were located in 19,361 genes with mapped VIR loci, were analyzed. Firstly, 26,291 pathogenic variants were excluded as they were located in VIRs with low coverage or in VIRs with zero length (i.e. regions between two variants in adjacent codons), since the latter had an evolutionary conservation score of zero. We then grouped all VIRs with high coverage in 2,935 genes with at least one pathogenic variant by their length in 5 bins $(1-5,6-10,11-15,16-20,21$ or more amino acids) (Fig. 1a), and counted the number and proportion of pathogenic variants that were located in VIRs in each bin (Fig. 1b). Missense and loss-of-function (LoF) variants were analyzed 
separately because LoF variant effect on a protein was expected to be less dependent on their location in the protein sequence. To compare pathogenic variant load in VIRs of different lengths, a "Pathogenic Variants per Amino Acid" metric was produced by dividing the number of pathogenic variants observed in VIRs in each bin by the summed length of all VIRs in that bin (Fig. 1c). To calculate 95\% confidence intervals for "Pathogenic Variants per Amino Acid" metric, the analysis was repeated 10,000 times on a randomly generated set of $50 \%$ of the 2,935 genes containing pathogenic variants (i.e. bootstraping) using resample method from python scikit-learn module ${ }^{22}$. To measure correlation between VIR length and evolutionary conservation, we grouped VIRs with high coverage in 5 bins (containing 1-5, 6-10, 11-15, 16-20, 21 or more amino acids; 1,938,102 VIRs in 18,491 genes in total) and displayed the distribution of VIR conservation scores using boxplots (Fig. 1d).

Gene Variation Intolerance Rank (GeVIR) calculation. To develop a gene-level metric based on 270 VIRs, relative weights of VIRs based on their length were first calculated. It was assumed that longer VIRs should have larger weights, since they might be less likely to be caused by random variant distribution. The number of VIRs (with high coverage) of each length were calculated and sorted based on length, in ascending order. We then calculated the weight $(W)$ of each length $(l)$ based on the ratio between all VIRs and the number of VIRs with length $I$ or longer:

$$
W_{l}=\frac{\sum_{i=0}^{l_{\max }} V I R_{i}}{\sum_{i=l}^{l_{\max }} V I R_{i}}
$$

For the number and weights of VIRs of each length, see Supplementary Tables 7 and 8 for autosomes and allosomes, respectively.

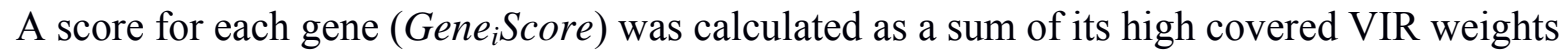
$\left(\mathrm{W}_{\mathrm{j}}\right)$ adjusted by their conservation $\left(G E R P_{j}\right)$ and normalized by the total number of regions in a gene 
variants or no evolutionary conservation data), were rounded to 1 or -1 to avoid extreme penalties in

282 VIR weight (W) adjustment due to multiplication:

$$
G E R P=\left\{\begin{array}{cc}
1, & \text { if } 0<G E R P<1 \\
-1, & \text { if }-1<G E R P<0 \\
G E R P, & \text { otherwise }
\end{array}\right.
$$

$$
\text { Gene }_{i} \text { Score }=\sum_{j}\left(W_{j} *{G E R P_{j}}\right) / \text { Nregions }_{i}
$$

284

285 VIRs with low coverage were counted in normalization $\left(\right.$ Nregions $\left._{i}\right)$ to avoid genes with a large 286 proportion ofVIRs with low coverage receiving high GeneScores due to the presence of a few highly 287 covered VIRs with large weights.

All 19,361 genes were sorted based on their GeneScores in descending order and percentiles calculated to generate a Gene Variation Intolerance Rank (GeVIR), where a lower rank indicates higher intolerance to variation (see Supplementary Table 2).

Disease and null and essential genes. Disease associated genes were downloaded from the Online Mendelian Inheritance in Man (OMIM) database ${ }^{5}$ (accessed November 2018). Only associations with confident phenotype mapping number (3) were included. Only genes that were associated exclusively with dominant (phenotype keywords: “autosomal dominant”) or recessive (phenotype keywords: "autosomal recessive") diseases were labeled as "AD" $(n=790)$ and "AR" $(n=1,585)$, respectively. Phenotypes which were marked as unconfirmed - “?”, non-diseases - “[]” or susceptibility - “ \{\}$"$ were excluded, as were genes that were associated with both AD and AR diseases. A set of genes with at least two different high-confidence LoF variants found in a homozygous state in at least one individual in ExAC ("Null”, $n=328)^{1}$, as well as cell essential $(n=663)$ and non-essential $(n=865)$ gene lists, 301 according to CRISPR/Cas studies ${ }^{15}$, were obtained from the macarthur-lab github repository 
302 (https://github.com/macarthur-lab/gene lists). Mouse essential genes were obtained from the MGI 303 database via MouseMine ${ }^{13,14}$ website (http://mousemine.org/). All genes from heterozygous mouse 304 models with a phenotype term containing the word "lethal" and that were linked to human orthologs 305 (http://www.informatics.jax.org/downloads/reports/HMD_HumanPhenotype.rpt) were exported $(n=$ 306 388). Note that genes which were not present in our list of 19,361 protein-coding genes were excluded 307 from all gene datasets.

GeVIR comparison with gnomAD gene constraint metrics. GeVIR performance was compared with three gnomAD gene constraint metrics: loss-of-function observed/expected upper bound fraction

311 (LOEUF) $)^{2}$, missense observed/expected upper bound fraction (referred to here as MOEUF, by analogy 312 with LOEUF) and Missense z-score ${ }^{3}$. LOEUF, MOEUF and Missense z-scores for the 19,361 genes 313 were obtained from gnomAD (https://gnomad.broadinstitute.org/downloads). To convert constraint 314 metrics into ranked lists, gene lists were sorted (LOEUF, MOEUF in ascending, Missense z-score in 315 descending order) and gene "weak" percentiles were computed, so that the most variation intolerant 316 genes have low percentiles. To investigate possible complementarity of GeVIR and LOEUF metrics a 317 “Combined" ranked list (VIRLoF) was created, where GeVIR and LOEUF ranks were summed for 318 each gene, the list resorted and percentiles recalculated. We evaluated ranked lists on six datasets: “AD”, "Mouse Heterozygous Lethal”, “Cell Essential”, “AR”, “Cell Non-essential” and "Null” (Table 3201 and Supplementary Fig. 2a-f). Area under the curve (AUC) method from python scikit-learn ${ }^{22}$ 321 module was used to measure performance on "AD”, "Mouse Heterozygous Lethal", "Cell Essential”, 322 "Cell Non-essential" and "Null" gene datasets. Genes from the "AR" dataset were expected to be 323 enriched in the middle of the ranked lists. To investigate this, we divided gene ranked lists into deciles 324 and calculated ratios between "AR" genes in each decile and all "AR" genes (Fig. 3a and 325 Supplementary Fig. 2f). Since a "good" ranked gene list should prioritize "AD" over "AR" genes, we 326 evaluated balance between "AD" and "AR" genes at each cumulative percentile X. We considered 
"AD" genes with percentiles $\leq \mathrm{X}$ as true positives (TP), "AR" genes with percentiles $\leq \mathrm{X}$ as false positives (FP), and "AD" genes with percentiles $>\mathrm{X}$ as false negatives $(\mathrm{FN})$. Based on these values, we calculated precision, recall and $\mathrm{F}_{1}$ score for "AD" gene class:

$$
\text { precision }=\frac{T P}{T P+F P} \text { recall }=\frac{T P}{T P+F N} F_{1} \text { score }=2 * \frac{\text { precision } * \text { recall }}{\text { precision }+ \text { recall }}
$$

332 F1 score could be used as a representative metric only for the first third of the genes (Supplementary 333 Fig. 2g) because, after some unknown threshold, "AR" genes had to be prioritized over "Null" genes, 334 which would result in higher numbers of FPs and thus lower precision and $\mathrm{F}_{1}$ scores. Therefore, peak $335 \quad F_{1}$ score was used for gene ranking method comparisons (Table 1). Performance was measured using $F_{1}$ 336 score of "AD" genes instead of accuracy or AUC, because there were $\sim 2$ times fewer "AD" than "AR" genes in the dataset. Similarity between GeVIR and other ranked gene lists was investigated by calculating the proportion of genes prioritized by both GeVIR and each other method at each cumulative percentile (Supplementary Fig. 2h). The relationship between gene ranks and protein length (based on the canonical transcript) was investigated by measuring Spearman's rank correlation coefficient $\left(\mathrm{SciPy}^{23}\right.$ package) and median protein length comparison at each decile for all ranked gene lists (Fig. 3b and Supplementary Figs. 2i and 3).

Note that the evaluation was performed on a dataset that included 1,009 genes containing extremely large or small numbers of variants (i.e. outliers) according to gnomAD gene constraint metrics $^{2}$, and genes without constraint metrics received the lowest ranks ( 1 gene in Missense z-score and 482 genes in LOEUF lists). However, we included outlier genes in GeVIR score calculations to provide metrics for a larger number of genes, since some of them are well known disease genes (e.g. CFTR associated with cystic fibrosis), and their presence did not decrease the performance of GeVIR.

349 Evaluation of gene scores on a dataset excluding outliers (18,352 genes) was also carried out and 350 showed the same overall trends (Supplementary Fig. 4). 
352 GeVIR and LOEUF comparison of most variation intolerant genes. We selected 2,989 out of 35319,361 genes $(\sim 15 \%)$ that had LOEUF $<0.35$ (a hard threshold suggested by the authors for most LoF 354 intolerant genes; L. Francioli, G. Tiao, K. Karczewski, M. Solomonson and N. Watts:

355 https://macarthurlab.org/2018/10/17/gnomad-v2-1/) and the same number of genes ranked at the top by 356 GeVIR and VIRLoF. Overlapping genes were investigated by using Venn diagrams (Fig. 4) to compare 357 all genes, "AD" and "AR" genes in the sets. To compare "prioritized gene similarity" with "method 358 performance", we also calculated precision, recall, and F1 score for the "AD" gene class. We then selected 1,317 genes present only in the GeVIR set, 1,317 genes present only in the LOEUF set, and 1,672 genes present in both sets, which were analyzed by DAVID $6.8^{16,17}$ for functional enrichment in Gene Ontology (GO) terms (biological process, cellular component, molecular function) and biological pathways (KEGG) (Supplementary Tables 5 and 6).

GeVIR comparison with Constrained Coding Regions (CCRs). Although no gene level metric was provided by the CCR study ${ }^{10}$, the authors compared it with gene constraint metrics by sorting genes based on the number of CCRs $\geq 95^{\text {th }}$ or greater percentile content within them. CCR data was downloaded from the browser (https://s3.us-east-2.amazonaws.com/ccrs/ccr.html) (accessed December 2018), and genes present in our study with at least one CCR at the $95^{\text {th }}$ percentile were selected $(7,000$ genes) and sorted, based on the number of CCRs, to create a ranked gene list. The same number of genes ranked at the top by GeVIR were selected, and the cumulative number of "AD" and "AR" genes as well as cumulative F1 score for "AD" gene class and protein length of prioritized genes were compared (Extended Data Fig. 1).

374 Life Sciences Reporting Summary. Further information on experimental design is available in the 375 Life Sciences Reporting Summary. 
378 Code availability. Code for calculating GeVIR/VIRLoF scores, data analysis and figures can be found

379 at https://github.com/gevirank/gevir. Computed GeVIR/VIRLoF scores are available in Supplementary

380 Table 2.

381

382

383

384

385

386

387

388

389

390

391

392

393

394

395

396

397

\section{Data Availability}

The GERP++ file can be found at http://mendel.stanford.edu/SidowLab/downloads/gerp/hg19.GERP_scores.tar.gz. The ClinVar files can be found at ftp://ftp.ncbi.nlm.nih.gov/pub/clinvar/tab delimited/variant summary.txt.gz and ftp://ftp.ncbi.nlm.nih.gov/pub/clinvar/tab delimited/var citations.txt. The Constrained Coding Regions (CCRs) files can be found at https://s3.us-east-

2.amazonaws.com/ccrs/ccrs/ccrs.autosomes.v2.20180420.bed.gz and https://s3.us-east2.amazonaws.com/ccrs/ccrs/ccrs.xchrom.v2.20180420.bed.gz. The OMIM genemap2.txt file can be found, after registration, at https://omim.org/downloads/. The gnomAD gene constraint metric file can be found at https://storage.googleapis.com/gnomadpublic/release/2.1.1/constraint/gnomad.v2.1.1.lof_metrics.by transcript.txt.bgz. The gnomAD exomes variants and coverage files can be found at https://storage.googleapis.com/gnomadpublic/release/2.0.2/vcf/exomes/gnomad.exomes.r2.0.2.sites.vcf.bgz and https://storage.googleapis.com/gnomadpublic/release/2.0.2/coverage/combined tars/gnomad.exomes.r2.0.2.coverage.all.tar, respectively. The gnomAD genomes variants files can be found at

https://storage.googleapis.com/gnomadpublic/release/2.0.2/vcf/genomes/gnomad.genomes.r2.0.2.sites.coding_only.chr1-22.vcf.bgz and https://storage.googleapis.com/gnomadpublic/release/2.0.2/vcf/genomes/gnomad.genomes.r2.0.2.sites.coding_only.chrX.vcf.bgz. The gnomAD genes, transcripts and exons files can be found at http://broadinstitute.org/ konradk/exac_browser/exac_browser.tar.gz. The Ensembl coding and peptide sequences from build GRCh37/hg19 can be found at https://grch37.ensembl.org/biomart/martview/ (Dataset: Human genes (GRCh37.p13); Attributes $\rightarrow$ Sequences $\rightarrow$ "Coding sequence" and "Peptide"). The homozygous Loss-of-function tolerant genes (i.e. Nulls) can be found at https://github.com/macarthurlab/gene lists/blob/master/lists/homozygous lof tolerant twohit.tsv. The cell essential and nonessential genes from CRISPR/Cas experiments can be found at https://github.com/macarthurlab/gene_lists/blob/master/lists/CEGv2_subset_universe.tsv and https://github.com/macarthurlab/gene lists/blob/master/lists/NEGv1 subset_universe.tsv, respectively. The mouse heterozygous lethal genes can be obtained from http://mousemine.org/ by quiring the database with following constraints: path="OntologyAnnotation.ontologyTerm" type="MPTerm"; path="OntologyAnnotation.subject" type="SequenceFeature"; path="OntologyAnnotation.evidence.baseAnnotations.subject" type="Genotype"; path="OntologyAnnotation.evidence.baseAnnotations.subject.zygosity" op="=" value="ht" code="B"; path="OntologyAnnotation.ontologyTerm.name" op="CONTAINS" value="lethal". The human-mouse ortholog mapping file can be found at 
420 http://www.informatics.jax.org/downloads/reports/HMD HumanPhenotype.rpt. The HGNC approved 421 gene symbols can be found at https://www.genenames.org/download/statistics-and-files/.

422

423

424

425

426

427

21. Yates, B. et al. Genenames.org: the HGNC and VGNC resources in 2017. Nucleic Acids Res. 45, D619-D625 (2017).

22. Pedregosa, F. et al. Scikit-learn: Machine Learning in Python. Journal of Machine Learning Research 12, 2825-2830 (2011).

23. Virtanen, P. et al. SciPy 1.0--Fundamental Algorithms for Scientific Computing in Python. arXiv:1907.10121 [physics] (2019). 
TCF4 (a)

TCF4

Charcot-Marie-Tooth disease, type 1C (AD)

LITAF

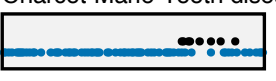

Metachromatic leukodystrophy (AR)

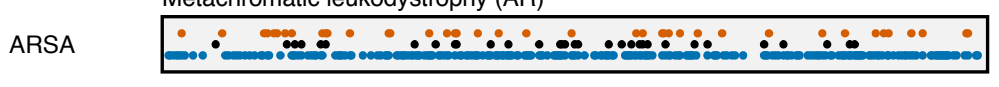

$\because \bullet \bullet \bullet \bullet \bullet \bullet \bullet \bullet$

Amyotrophic lateral sclerosis, with or without Frontotemporal lobar degeneration (AD)

TARDBP

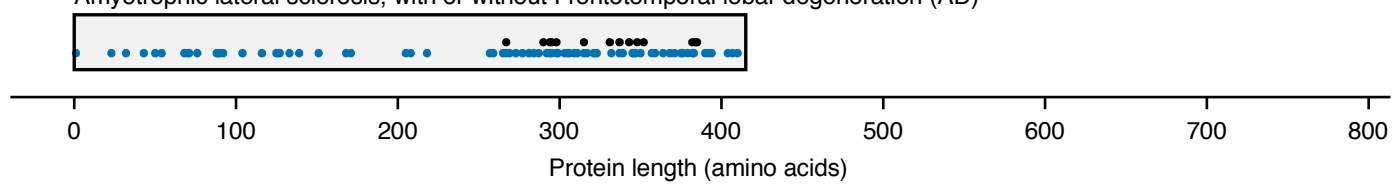

$\boldsymbol{b}$

VIR's coverage (mean of 11 nucleotides)

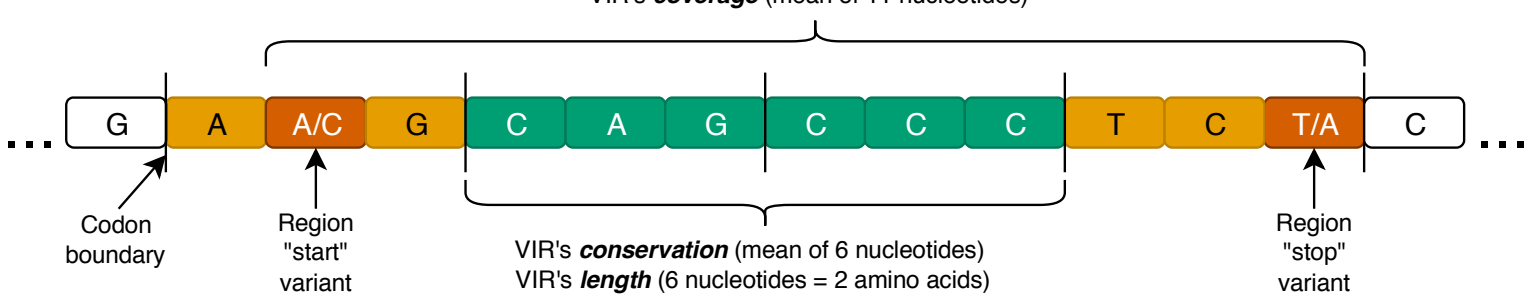

c

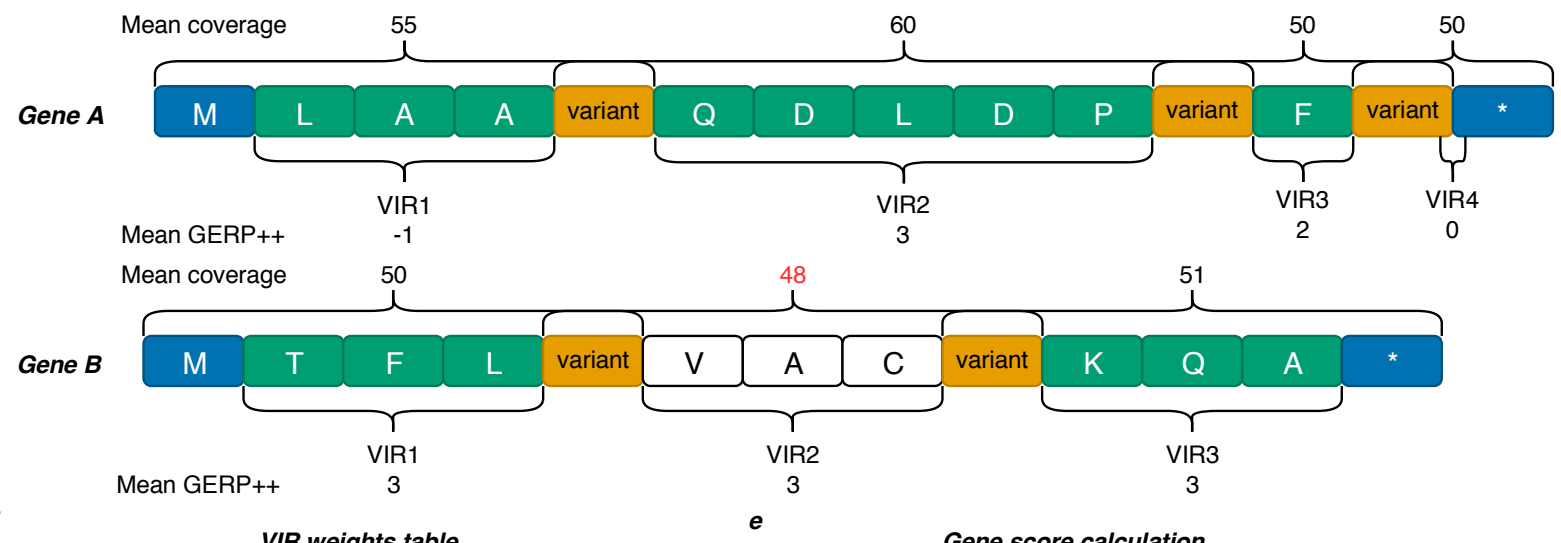

\begin{tabular}{|c|c|c|}
\hline Length & Number $^{*}$ & Weight $^{\star *}$ \\
\hline 0 & 1 & $6 /(1+1+3+1)=1$ \\
\hline 1 & 1 & $6 /(1+3+1)=1.2$ \\
\hline 3 & 3 & $6 /(3+1)=1.5$ \\
\hline 5 & 1 & $6 / 1=6$ \\
\hline
\end{tabular}

* VIRs with mean coverage $<50$ (45 for $X$ and $Y$ chromosomes, analysed separately) are not counted.

** Weight is calculated as total number of VIRs divided by number of VIRs with this length or less

Gene $_{i}$ Score $=\sum_{j}\left(W_{j} * G E R P_{j}\right)$ Nregions $_{i}$

$G E R P=\left\{\begin{array}{cl}1, & \text { if } 0<G E R P<1 \\ -1, & \text { if }-1<G E R P<0 \\ G E R P, & \text { otherwise }\end{array}\right.$

VIRs $s_{i}$ include low coverage (in white)

Gene $_{a}$ Score $=\frac{1.5 \times(-1)+6 \times 3+1.2 \times 2+1 \times 0}{4}=4.725$

Gene $_{b}$ Score $=\frac{1.5 \times 3+1.5 \times 3}{3}=1.5$ 

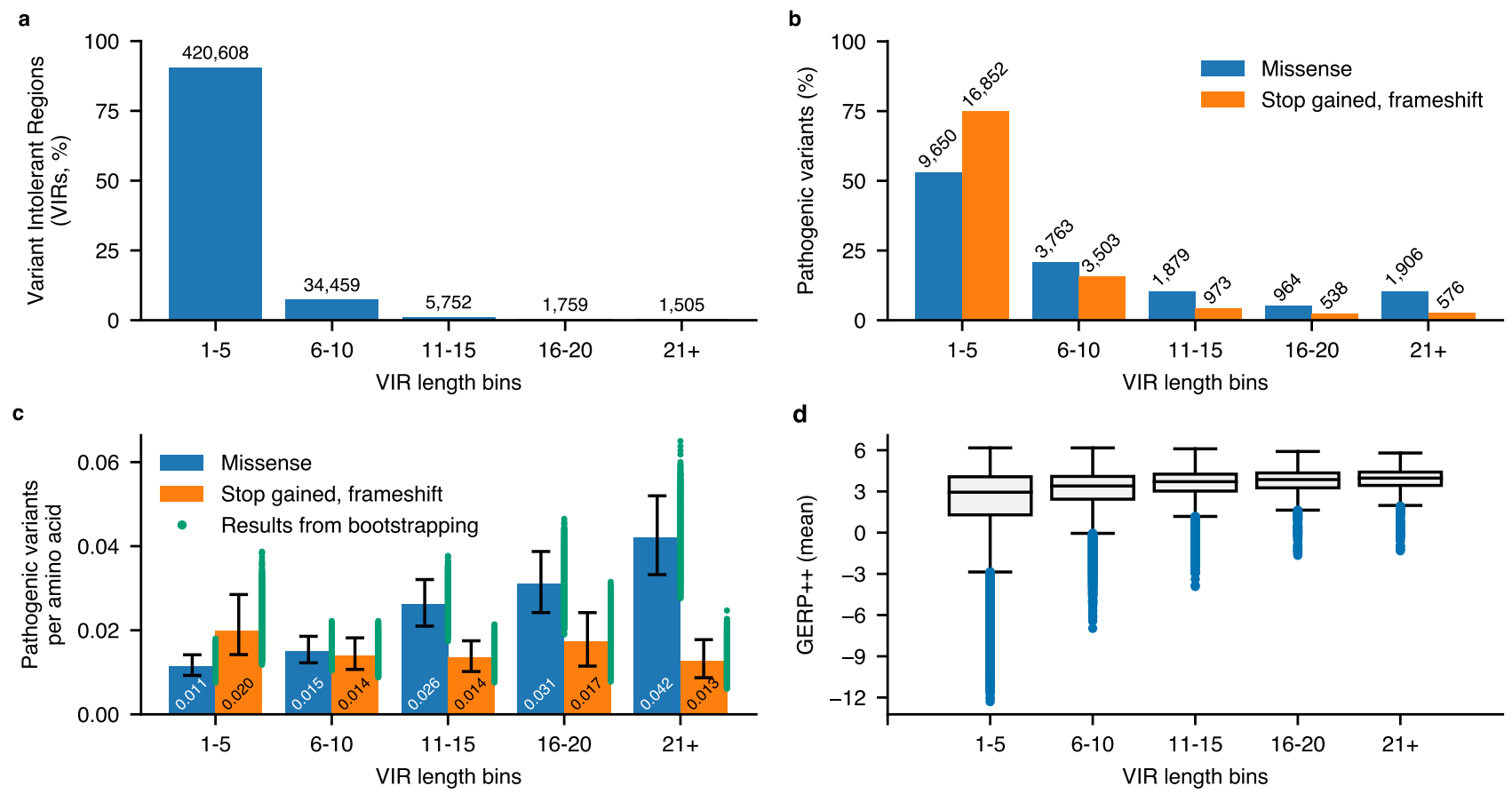
a

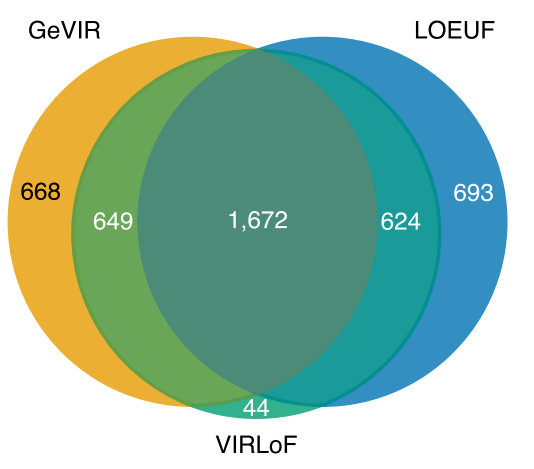

c

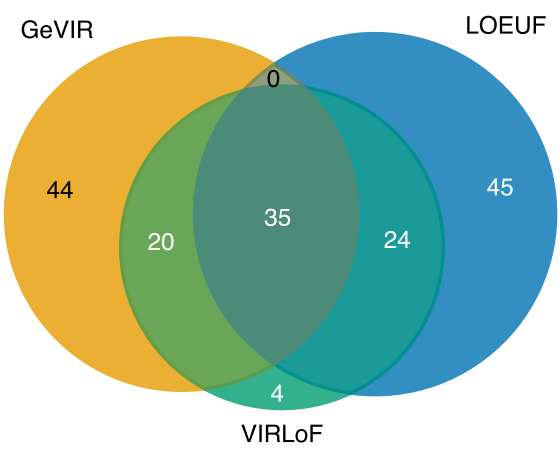

b

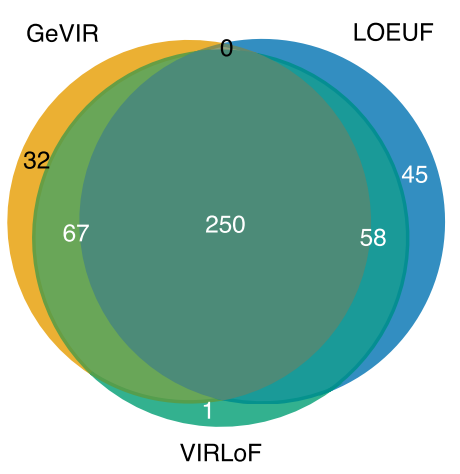

d

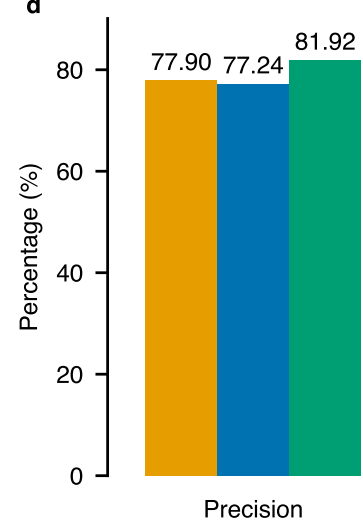

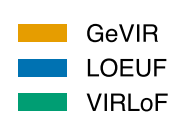

$56.3856 .62^{60.21}$

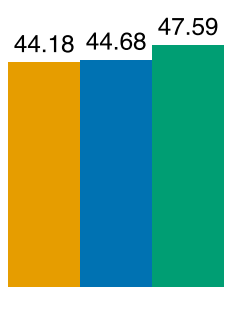

Recall 
